\title{
"SOBREVIVÊNCIA" NO EMPREGO DOS TRABALHADORES DE ENFERMAGEM EM UMA INSTITUIÇÃO HOSPITALAR PÚBLICA
}

\author{
Maria Luiza Anselmi \\ Geraldo Garcia Duarte ${ }^{2}$ \\ Emília Luigia Saporiti Angerami ${ }^{3}$
}

Anselmi ML, Duarte GG, Angerami ELS. "Sobrevivência" no emprego dos trabalhadores de enfermagem em uma instituição hospitalar pública. Rev Latino-am Enfermagem 2001 julho; 9(4):13-8.

O presente trabalho estima o tempo de "sobrevivência" no emprego dos trabalhadores de enfermagem, após sua admissão em um hospital público, como um dos indicadores de rotatividade. Utilizou-se a técnica de Tábua de vida de seguimento para calcular a probabilidade de sobrevivência no emprego por $X$ anos para cada uma das categorias de trabalhadores. Os resultados apontaram, para o período estudado, uma rotatividade acentuada dessa força de trabalho. As categorias, auxiliar de enfermagem e enfermeiro apresentaram baixa estabilidade no emprego enquanto a categoria técnico de enfermagem foi a mais estável.

PALAVRAS CHAVE: emprego, recursos humanos de enfermagem

\section{EMPLOYMENT SURVIVAL OF NURSING WORKERS IN A PUBLIC HOSPITAL}

This study aimed at estimating the employment "survival" time of nursing workers after their admission to a public hospital as a turnover index. The Life Table method was used in order to calculate the employment survival probability by $X$ years for each one of the categories of workers. The results showed an accentuated turnover of the work force in the studied period. The categories nursing auxiliary and nurse presented low stability in employment while the category nursing technician was more stable.

KEY WORDS: employment, nursing human resourses, turnover

\section{SOBREVIVENCIA EN EL EMPLEO DE LOS TRABAJADORES DE ENFERMERÍA EN UNA INSTITUCIÓN HOSPITALARIA PÚBLICA}

El presente trabajo mide el tiempo de "sobrevivencia" en el empleo de los trabajadores de enfermería, después de su admisión en un hospital público, como uno de los indicadores de rotación. Se utilizó la técnica de la Tabla de vida de seguimiento para calcular la probabilidad de sobrevivencia en el empleo por $X$ años para cada una de las categorías de trabajadores. Los resultados mostraron, para el periodo estudiado, una rotación acentuada de esa fuerza de trabajo. Las categorías, auxiliar de enfermería y enfermero presentaron baja estabilidad en el empleo mientras que la categoría técnico de enfermería fue la más estable.

PALABRAS CLAVES: empleo, recursos humanos en enfermería

\footnotetext{
${ }^{1}$ Enfermeira, Professor Doutor, e-mail: anselmi@eerp.usp.br ; ${ }^{2}$ Matemático, Professor Adjunto; ${ }^{3}$ Enfermeira, Professor Titular, da Escola de Enfermagem de Ribeirão Preto da Universidade de São Paulo, Centro Colaborador da OMS para o desenvolvimento da pesquisa em enfermagem
} 


\section{INTRODUÇÃO}

Até a década de 70, o emprego em saúde vinha crescendo numa taxa média de $13 \%$ ao ano mas, no final desse período, passou a ser de 8,6\% ao ano. Entre 1980 e 1984, o número de postos de trabalho em saúde cresceu em torno de $4 \%$ e, na presente década, 0 emprego formal em serviços de saúde apresenta uma média anual geométrica de crescimento de aproximadamente, $2 \%$ ao ano ${ }^{(1)}$.

Segundo estimativas baseadas em dados da Relação Anual de Informações Sociais (RAIS) e do Cadastro Geral de Empregados e Desempregados (CAGED) do Ministério do Trabalho, em agosto de 1998, existiam cerca de 2,15 milhões de pessoas empregadas em atividades de saúde, o que representava cerca de 8,7\% do total de empregos existentes na economia formal do país. No segmento de atividades de atendimento hospitalar, de urgências e emergências, existiam em julho de 1998, aproximadamente, 10.000 estabelecimentos, os quais detinham cerca de 650.000 empregos. No que se refere à enfermagem, cerca de $80 \%$ dos empregos de enfermeira, $90 \%$ dos empregos de auxiliar de enfermagem e $87 \%$ dos de atendentes de enfermagem estavam alocados neste segmento ${ }^{(2)}$. Portanto, a rede hospitalar constitui, ainda, um polo expressivo de demanda de trabalhadores, principalmente da área da enfermagem.

A dinâmica do mercado de trabalho em saúde caracterizase pela oferta e demanda de força de trabalho cuja configuração dáse em função de um conjunto de determinações políticas, econômicas e institucionais. Além destes elementos (oferta e demanda), outro movimento que se faz presente nessa dinâmica é a movimentação da força de trabalho no mercado de trabalho. Tal movimentação, caracterizada pela rotatividade é, segundo o Departamento Intersindical de Estatística e Estudos Sócio-econômicos" ${ }^{(3)}$, "a medida do número de trabalhadores que passa pelos postos de trabalho de uma seção, empresa, setor ou ramo, num determinado período de tempo". Este conceito está intimamente relacionado à idéia de reposição, substituição ou rodízio da força de trabalho. À medida em que estas reposições intensificam-se, mediante as saídas do emprego, é possível observar sérias repercussões no que se refere à eficiência, à eficácia, à efetividade e por conseqüência, à qualidade dos serviços prestados, seja no âmbito mais restrito do processo de trabalho institucional seja no segmento da produção onde a rotatividade ocorre.

Nos E.U.A., a carência quantitativa de enfermeiras no mercado e, a alta rotatividade no emprego motivaram a realização dos primeiros estudos sobre o assunto. Uma vasta produção científica acerca da rotatividade é encontrada na literatura norte americana que, desde os anos 50, vem desenvolvendo investigações no sentido de conhecer melhor este fenômeno no setor saúde, em especial, na área de enfermagem. Atualmente, a rotatividade permanece ainda como temática de interesse dos pesquisadores, pelas sérias implicações financeiras que provoca no desenvolvimento das atividades organizacionais, principalmente no que se refere aos custos, à diminuição da produtividade e da qualidade dos serviços ${ }^{(4-}$ 10)

No Brasil, ainda hoje, são poucos os estudos acerca da rotatividade do pessoal de enfermagem, principalmente no que diz respeito à sua mensuração embora, em vários trabalhos, esse movimento seja referido como fortemente presente nos serviços de saúde ${ }^{(11-17)}$

A movimentação dos trabalhadores de enfermagem no mercado de trabalho, segundo entradas, saídas e tempo de permanência no emprego, constitui elemento relevante no processo gerencial dos serviços. Uma força de trabalho estável e qualificada além de sustentar o processo de cuidar, cria também possibilidades efetivas de garantia da qualidade dos serviços ofertados. 0 estudo ora apresentado tem por objetivo estimar o tempo de "sobrevivência" no emprego de um grupo de trabalhadores de enfermagem após sua admissão em um hospital público, colocando assim, em evidência, o grau de estabilidade/instabilidade (e portanto, da rotatividade) dessa força de trabalho em uma instituição específica.

\section{METODOLOGIA}

O estudo foi realizado no Hospital das Clínicas da Faculdade de Medicina de Ribeirão Preto, Universidade de São Paulo (HCFMRPUSP), localizado no interior do estado de São Paulo. Trata-se de um centro de referência na atenção à saúde, não só para o estado mas para o país. As ações desenvolvidas pela instituição envolvem o ensino, a pesquisa e a assistência médica integral em ambulatórios e leitos hospitalares abrangendo praticamente todas as especialidades médicas. Em dezembro de 1994, o hospital contava com 606 leitos e 1433 funcionários alocados na Divisão de Enfermagem.

O período do estudo foi de 5 anos, qual seja, de 01/01/ 1990 a 31/12/1994. A população do estudo constituiu-se de todos os trabalhadores de enfermagem (enfermeiro, técnico e auxiliar de enfermagem) admitidos ao longo do período estudado e lotados na Divisão de Enfermagem. Com a autorização da direção do hospital, estes trabalhadores foram acompanhados quanto à data de admissão, data de desligamento e tempo de permanência no emprego. As informações foram coletadas a partir de consulta ao Cadastro Geral de Empregados e Desempregados (CAGED) - Lei n 4923/65 do Ministério do Trabalho, preenchido mensalmente pelo Centro de Recursos Humanos da Instituição. Tratando-se de documento público, 
houve facilidade de acesso aos dados e pelo fato do Ministério Público exigir, de todas as instituições empregadoras, o repasse periódico destas informações, assegura-se a fidedignidade dos dados coletados.

Para estimar a probabilidade de "sobrevivência" no emprego por $\mathrm{X}$ anos, para cada uma das categorias de trabalhadores estudadas, utilizou-se a Técnica de Tábua de vida de seguimento, a qual tem sido adotada para realização de "estudos de sobrevida" ou "estudos de seguimento" (follow-up) permitindo estimar para um determinado grupo (coorte), a probabilidade de sobreviver por um número $\mathrm{X}$ de anos, após o diagnóstico de uma determinada doença crônica, ou em comparar probabilidades de sobrevivência após tratamentos específicos ${ }^{(18-21)}$. Em síntese, dada uma coorte com todos os indivíduos num Estado $\left(\mathrm{E}_{0}\right)$, a técnica permite calcular a porcentagem dessa coorte ainda no $E_{0}$ após um tempo $\mathbf{x}$ de observação, admitindo-se perdas de observação(censura). Esta porcentagem estima a probabilidade de 0 indivíduo permanecer no Estado $\mathrm{E}_{0}$ após $\mathbf{x}$ unidades de tempo.

Tempo de sobrevida é definido como "...a quantidade de tempo que um indivíduo permanece em um estado bem definido (vivo, internado, doente, são), a partir de um evento perfeitamente caracterizado (nascimento, admissão no hospital, diagnóstico, alta clínica), até um segundo evento, também exatamente marcado (óbito, demissão, alta hospitalar, recidiva) ${ }^{\text {(18) }}$. Desta forma, tem-se dois eventos e um tempo medido entre eles.

A vantagem da utilização de tal técnica para estimar a probabilidade de sobrevivência de pacientes advém do fato de levar em consideração a contribuição parcial dos indivíduos que não permaneceram todo o tempo em estudo (as chamadas perdas de observação ou censura).

Com base nos conceitos propostos pela Técnica Tábua de vida de seguimento, estabeleceu-se uma analogia com a questão da rotatividade. Para estimar a probabilidade de "sobrevivência" no emprego, após a data de admissão, de um grupo de trabalhadores de enfermagem, por um número $X$ de anos, considerou-se a data de admissão do trabalhador no emprego como o evento perfeitamente caracterizado $\left(\mathrm{E}_{0}\right)$; o desligamento do emprego como o evento exatamente marcado $\left(E_{1}\right)$. Uma vez que o interesse era conhecer quanto tempo o trabalhador, por iniciativa própria, permanecia no emprego, definiu-se como perdas de observação ou censura, as saídas provocadas por iniciativa do empregador e ainda, os indivíduos falecidos no período. Considerou-se como "sobrevida" no estudo, a quantidade de tempo que cada grupo de trabalhador, após a admissão, permaneceu empregado na instituição (estado bem definido). Neste sentido, a Tábua de vida de seguimento pode ser considerada um indicador específico e mais refinado para mensurar e avaliar a rotatividade.

\section{RESULTADOS E DISCUSSÃO}

Desde 01/01/1990 foram acompanhados 566 trabalhadores de enfermagem (314 auxiliares de enfermagem, 27 técnicos de enfermagem e 225 enfermeiros) em termos de admissão, demissão e tempo de permanência na instituição estudada. Ao final do período (31/12/1994), desse grupo seguido, encontravam-se empregados na instituição 322 trabalhadores sendo: 131 enfermeiros, 169 auxiliares e 22 técnicos de enfermagem.

A Tabela 1 apresenta a Tábua de vida de seguimento de todos os trabalhadores acompanhados no período. Verifica-se que a probabilidade desse grupo permanecer no emprego após 5 anos é de $26,17 \%$, ou de outra forma, dos 566 trabalhadores admitidos no Hospital no período de janeiro/90 a dezembro/94 apenas 26,17\% "sobreviveram" ou seja continuam empregados. Estes dados apontam para uma forte renovação da força de trabalho na instituição ao longo destes 5 anos.

Pela Tabela 2 temos a Tábua de vida de seguimento aplicada à categoria enfermeiro. Dos 225 enfermeiros seguidos, apenas $27,8 \%$ têm probabilidade de permanecer no emprego após 5 anos. Na Tabela 3, dos 314 auxiliares de enfermagem acompanhados, 23,5\% têm probabilidade de manter o vínculo empregatício neste hospital. Quanto aos técnicos de enfermagem, 89,5\% apresentam probabilidade de continuar no emprego após 5 anos da sua admissão na instituição (Tabela 4).

Tabela 1 - Tábua de vida de seguimento dos trabalhadores de enfermagem* após admissão no HCFMRP-USP - 01/1990 a 12/1994

\begin{tabular}{|c|c|c|c|c|c|c|c|c|c|}
\hline Período & $\begin{array}{r}x \mid-x+1 \\
\text { anos de } \\
\text { observaçẫo }\end{array}$ & $\begin{array}{l}\text { li } \\
\text { no início do } \\
\text { período }\end{array}$ & $\begin{array}{c}\mathrm{Di} \\
\text { de sligados } \\
\text { durante o período }\end{array}$ & $\begin{array}{c}\text { Vot* } \\
\text { perdidos ou } \\
\text { desligados por } \\
\text { outras causas }\end{array}$ & $\begin{array}{c}\text { Ioli } \\
\text { finalizaram o } \\
\text { último período } \\
\text { empregados }\end{array}$ & $\begin{array}{c}\mathrm{Yi} \\
\text { expostos ao } \\
\text { desligamento }\end{array}$ & $\begin{array}{c}\text { qi } \\
\text { proporçẫo } \\
\text { desligados } \\
\text { no período }\end{array}$ & $\begin{array}{c}\text { pi } \\
\text { proporçẫo que } \\
\text { continua } \\
\text { empregado }\end{array}$ & $\begin{array}{c}\mathrm{Pi} \\
\text { proporçẫo dos que } \\
\text { permanecem no } \\
\text { emprego a partir do } \\
\text { início da } \\
\text { observaçẫo }\end{array}$ \\
\hline 1 & $0 \mid-1$ & 566 & 102 & 23 & 178 & 465,5 & 0,2191192 & 0,7808808 & $7809 \%$ \\
\hline 2 & $1 \mid-2$ & 263 & 48 & 2 & 32 & 246 & 0,1951219 & 0,8048781 & $62,85 \%$ \\
\hline 3 & $2 \mid-3$ & 181 & 35 & - & 29 & 166,5 & 0,2102102 & 0,7897898 & $49,64 \%$ \\
\hline 4 & $3 \mid-4$ & 117 & 24 & - & 37 & 98,5 & 0,2436548 & 0,7563452 & $37,54 \%$ \\
\hline 5 & $4 \mid-5$ & 56 & 10 & - & 46 & 33 & 0,3030303 & 0,6969697 & $26,17 \%$ \\
\hline
\end{tabular}

* Auxiliar de enfermagem, Técnico de Enfermagem e Enfermeiro

${ }^{* *}$ No estudo considerou-se como perdidos aqueles trabalhadores que foram desligados pela instituição (seja por justa causa ou sem justa causa), e ainda aqueles que faleceram no período 
Tabela 2 - Tábua de vida de seguimento dos enfermeiros após admissão no HCFMRP-USP, 01/1990 a 12/1994

\begin{tabular}{|c|c|c|c|c|c|c|c|c|c|}
\hline Período & $\begin{array}{c}x \mid-x+1 \\
\text { anos de } \\
\text { observaçỗo }\end{array}$ & $\begin{array}{c}\text { li } \\
\text { no início } \\
\text { do período }\end{array}$ & $\begin{array}{c}\mathrm{Di} \\
\text { desligados } \\
\text { durante o período }\end{array}$ & $\begin{array}{c}\text { V/t* } \\
\text { perdidos ou } \\
\text { desligados por } \\
\text { outras causas }\end{array}$ & $\begin{array}{c}\text { Muli } \\
\text { finalizaram o } \\
\text { cultimo período } \\
\text { empregados }\end{array}$ & $\begin{array}{c}\mathrm{Yi} \\
\text { expostos ao } \\
\text { desligamento }\end{array}$ & $\begin{array}{c}\text { qi } \\
\text { proporçẫo } \\
\text { desligados } \\
\text { no período }\end{array}$ & $\begin{array}{c}\text { pi } \\
\text { proporçẫo } \\
\text { que continua } \\
\text { empregado }\end{array}$ & $\begin{array}{c}\mathrm{Pi} \\
\text { proporçẫo dos } \\
\text { que permanecem } \\
\text { no emprego a } \\
\text { partir do inicio da } \\
\text { observaçẫo }\end{array}$ \\
\hline 1 & $01-1$ & 225 & 41 & 8 & 70 & 186 & 0,2204301 & 0,7795699 & $7796 \%$ \\
\hline 2 & $1 \mid-2$ & 106 & 14 & 1 & 13 & 99 & 0.1414141 & 0,8585859 & $6694 \%$ \\
\hline 3 & $2 \mid-3$ & 78 & 15 & - & 11 & 72,5 & 0,2068965 & 0,7931035 & $5309 \%$ \\
\hline 4 & $3 \mid-4$ & 52 & 11 & - & 18 & 43 & 0,2558139 & 0,744187 & $3950 \%$ \\
\hline 5 & $4 \mid-5$ & 23 & 4 & - & 19 & 13,5 & 0,2962962 & 0,7037038 & $27,0 \%$ \\
\hline
\end{tabular}

Tabela 3 - Tábua de vida de seguimento dos auxiliares de enfermagem após admissão no HCFMRP-USP, 01/1990 a 12/1994

\begin{tabular}{|c|c|c|c|c|c|c|c|c|c|}
\hline Peŕodo & $\begin{array}{c}x \mid-x+1 \\
\text { anos de } \\
\text { okservaçấo }\end{array}$ & $\begin{array}{c}\text { li } \\
\text { no início } \\
\text { do período }\end{array}$ & $\begin{array}{c}\mathrm{Di} \\
\text { desligados } \\
\text { durante o período }\end{array}$ & $\begin{array}{c}\text { W/ } \\
\text { perdidos ou } \\
\text { desligados por } \\
\text { outras causas }\end{array}$ & $\begin{array}{c}\text { Muli } \\
\text { finalizaram o } \\
\text { cultimo período } \\
\text { empregados }\end{array}$ & $\begin{array}{c}\text { Pi } \\
\text { expostos ao } \\
\text { desligamento }\end{array}$ & $\begin{array}{c}\text { qi } \\
\text { proporçẫo } \\
\text { desligados } \\
\text { no período }\end{array}$ & $\begin{array}{c}\text { pi } \\
\text { proporçã̃o } \\
\text { que continua } \\
\text { empregado }\end{array}$ & $\begin{array}{c}\mathrm{Pi} \\
\text { proporçẫo dos } \\
\text { que permanecem } \\
\text { no emprego a } \\
\text { partir do início da } \\
\text { observaçẫo }\end{array}$ \\
\hline $\begin{array}{l}1 \\
2 \\
3 \\
4 \\
5\end{array}$ & $\begin{array}{l}0 \mid-1 \\
1 \mid-2 \\
2 \mid-3 \\
3 \mid-4 \\
4 \mid-5\end{array}$ & $\begin{array}{l}314 \\
148 \\
101 \\
63 \\
31\end{array}$ & $\begin{array}{l}59 \\
34 \\
20 \\
13 \\
6\end{array}$ & $\begin{array}{c}12 \\
1 \\
- \\
- \\
-\end{array}$ & $\begin{array}{l}95 \\
12 \\
18 \\
19 \\
25\end{array}$ & $\begin{array}{l}260,5 \\
141,5 \\
92 \\
53,5 \\
18,5\end{array}$ & $\begin{array}{l}0,2264875 \\
0,2402826 \\
0,2173913 \\
0,2429906 \\
0,3243243\end{array}$ & $\begin{array}{l}0,7735125 \\
0,7597174 \\
0,7826087 \\
0,7570094 \\
0,6756757\end{array}$ & $\begin{array}{l}77,35 \% \\
58,76 \% \\
45,98 \% \\
34,31 \% \\
23,52 \%\end{array}$ \\
\hline
\end{tabular}

Tabela 4 - Tábua de vida de seguimento dos técnicos de enfermagem após admissão no HCFMRP-USP, 01/1990 a 12/1994

\begin{tabular}{|c|c|c|c|c|c|c|c|c|c|}
\hline Perído & $\begin{array}{c}x \mid-x+1 \\
\text { anos de } \\
\text { observoçẫo }\end{array}$ & $\begin{array}{c}\text { li } \\
\text { no início } \\
\text { do período }\end{array}$ & $\begin{array}{c}\mathrm{Di} \\
\text { desligados } \\
\text { durante o periodo }\end{array}$ & $\begin{array}{c}\text { V/t* } \\
\text { perdidos ou } \\
\text { desligados por } \\
\text { outras causas }\end{array}$ & $\begin{array}{c}\text { Moli } \\
\text { finalizaram o } \\
\text { último período } \\
\text { empregados }\end{array}$ & $\begin{array}{c}\mathrm{Yi} \\
\text { expostos ao } \\
\text { desligamento }\end{array}$ & $\begin{array}{c}\text { qi } \\
\text { proporçẫo } \\
\text { desligados } \\
\text { no período }\end{array}$ & $\begin{array}{c}\text { pi } \\
\text { proporção } \\
\text { que continua } \\
\text { empregado }\end{array}$ & $\begin{array}{c}\mathrm{Pi} \\
\text { proporçẫo dos } \\
\text { que permanecem } \\
\text { no emprego a } \\
\text { partir do início da } \\
\text { observaçấo }\end{array}$ \\
\hline 1 & $0 \mid-1$ & 27 & 2 & 3 & 13 & 19 & 0,1052631 & 0,8947369 & $89,47 \%$ \\
\hline 2 & $1 \mid-2$ & 9 & - & - & 7 & 5,5 & 0 & 0,8947369 & $89,47 \%$ \\
\hline 3 & $2 \mid-3$ & 2 & - & - & - & 5 & 0 & 0,8947369 & $89,47 \%$ \\
\hline 4 & $3 j-4$ & 2 & - & - & . & 2,5 & 0 & 0,8947369 & $89,47 \%$ \\
\hline 5 & $4 \mid-5$ & 2 & - & - & 2 & 1 & 0 & 0,8947369 & $89,47 \%$ \\
\hline
\end{tabular}

As probabilidades encontradas para a "sobrevivência" no emprego após os 5 anos, evidenciam que as categorias enfermeiro e auxiliar de enfermagem apresentam baixa estabilidade no emprego, enquanto a categoria de maior estabilidade foi a de técnico de enfermagem. 0 grau de estabilidade/instabilidade da força de trabalho no emprego constitui um indicador do nível de rotatividade presente na organização ${ }^{(22)}$ e, neste sentido, pode-se afirmar que houve, durante o período analisado, uma rotatividade elevada de enfermeiros e auxiliares de enfermagem e, uma baixa movimentação no trabalho por parte dos técnicos de enfermagem.

Embora neste estudo não se tenha aprofundado sobre as razões da acentuada rotatividade para as categorias enfermeiro e auxiliar de enfermagem, pelos estudos realizados anteriormente nos hospitais do Município, incluindo o HCFMRP-USP ${ }^{(14,15)}$, e o acompanhamento da dinâmica do mercado de trabalho neste espaço, é possível apontar para alguns elementos que podem ter provocado essa movimentação da força de trabalho, entre eles:

- A reorganização dos serviços municipais de saúde, em especial, da rede básica que propiciou, nos últimos anos, uma expansão acelerada das unidades de saúde trazendo consigo um crescimento significativo no quantitativo de pessoal de enfermagem. Com o convênio SUDS firmado com o Município em 1987, ocorreu ampliação física e funcional da rede que passou a contar com 19 Unidades Básicas de Saúde (U.B.S.), 3 Pronto-Socorros e um quadro de pessoal de enfermagem de 40 atendentes, 25 auxiliares e 7 enfermeiros. Em 1997, com a implementação do processo de municipalização existiam 5 Unidades Básicas Distritais de Saúde, 26 U.B.S., 2 Núcleos Assistência Psico-Social, 1 Núcleo de Gestão Assistencial e 1 Ambulatório Regional de Saúde Mental. Este conjunto de serviços alocavam 60 atendentes, 494 auxiliares de enfermagem, 66 técnicos de enfermagem e 154 enfermeiros ${ }^{(23)}$. Diante destes fatos, é possível supor que esta ampliação no quantitativo de trabalhadores de 
enfermagem tenha se dado às custas dos hospitais, ou seja, que um grande contingente de pessoal tenha migrado da rede hospitalar para os serviços de saúde do Município.

- Na presente década, as políticas econômicas delineadas para o país(de cunho privatizante) influíram nas políticas traçadas para 0 setor saúde. Observa-se ao longo dos últimos anos um forte sucateamento da rede hospitalar pública evidenciado através da precariedade das condições de trabalho (horários e turnos de trabalho, salários, volume de atividades desenvolvidas) a que está submetida a força de trabalho nela alocada. Por outro lado, à medida em que a rede básica do Município reorganiza-se, ampliam-se também as perspectivas de trabalho, uma vez que neste âmbito, os horários e turnos de trabalho são mais flexíveis pois não existe o sistema de plantão noturno e a carga horária semanal é menor.

- Outro elemento a destacar, na esfera do gerenciamento de Recursos Humanos, diz respeito ao processo de implantação do plano de carreira, cargos e salários para cumprir as exigências legais de repasse de verbas do Fundo Nacional de Saúde para o Fundo Municipal de Saúde. Nos anos 90 , a remuneração dos trabalhadores de enfermagem na rede municipal tem se mantido superior à da rede hospitalar pública (e privada também) não só no que se refere ao salário base mas também porque tem agregado, a essa remuneração, o pagamento por produtividade. Estes fatos podem ser considerados como elementos atrativos para a saída do trabalhador do hospital para a rede básica.

\section{REFERÊNCIAS BIBLIOGRÁFICAS}

1. Girardi SN. Sinais de mercado e regulação institucional do trabalho em saúde nos anos 90. Brasília (DF):CGDRH-SUS; 1996.

2. Girardi SN. Mercado de trabalho em enfermagem no Brasil. Brasilia (DF): Secretaria de Políticas de Saúde CGDRH-SUS Ministério da Saúde; 1998. (Dossiê).

3. Departamento Intersindical de Estatística e Estudos Sócioeconômicos (BR). Rotatividade: conceito e determinantes (Primeira parte). São Paulo (SP): Bol Dieese 1988; 7:7-19.

4. Prescott $P A$, Bowen SA. Controlling nursing turnover. Nurs Manage 1987 June; 18(6):60-6.

5. Prestholdt PH, Lane IM, Mathews RC. Predicting staff nurse turnover. Nurs Outlook 1988 May-June; 36(3):145-7.

6. Jones CB. Staff nurse turnover costs: part I, A conceptual model. J Nurs Administration 1990 April; 20(4):18-22.

7. Cavanagh SJ. Predictors of nursing staff turnover. J Adv Nurs 1990 March; 15(3):373-80.

8. MacRobert M, Schmele JA, Henson R. An analysis of job morale factors of community health nurses who report a low turnover rate the research. J Nurs Administration 1993 June; 23(6):22-7.

\section{CONSIDERAÇÕES FINAIS}

Os processos assistenciais desenvolvidos pelos trabalhadores de enfermagem (auxiliar, técnico e enfermeiro) em uma instituição hospitalar podem ser assegurados quantitativa e qualitativamente à medida em que esse grupo de pessoas apresenta certo grau de estabilidade no emprego. Este estudo verificou, através de uma técnica específica (Tábua de vida de seguimento) que em um período de 5 anos, a força de trabalho em enfermagem da instituição estudada mostrou elevado grau de instabilidade. Embora não se aprofundando na identificação das suas causas, é possível apontar o processo de reorganização dos serviços de saúde do município nesta última década, como um dos determinantes da rotatividade acentuada desse grupo de trabalhadores.

Outros estudos se fazem necessários no sentido de ampliar e aprofundar o conhecimento acerca desse fenômeno, em especial, sobre os motivos de sua ocorrência cuja intensidade pode estar afetando a qualidade da assistência de enfermagem prestada na instituição em questão. Deste modo, entende-se que será possível repensar algumas estratégias na esfera do gerenciamento de Recursos Humanos, principalmente no que se refere à perspectiva de maior retenção desses trabalhadores no emprego.

9. Davidson H, Folcarelli PH, Crawford S, Duprat LJ, Clifford JC. The effects of health care reforms on job satisfaction and voluntary turnover among hospital-based nurses. Med Care 1997; 35(6):634-45.

10. Borda RG, Norman IJ. Factors influencing turnover and absence of nurses: a research review. Int J Nurs Stud 1997; 34 (6):385-94.

11. Costenaro OMV, Ribeiro AM, Tognini DL, Campoleoni ST. Rotatividade de pessoal: causas, conseqüências e propostas. Rev Paul Enfermagem 1987 janeiro/dezembro; 7(1):40-3.

12. Anselmi ML. Por que os enfermeiros deixam o emprego: estudo num Hospital-Escola. Rev Esc Enfermagem USP 1990 agosto; 24(2):237-50.

13. Anselmi ML, Angerami ELS, Infante AM. A rotatividade dos enfermeiros de um Hospital-Escola. Rev Educ Médica y Salud 1990; 24(4):452-61.

14. Aquino KS. Fatores geradores da rotatividade dos enfermeiros de um hospital geral governamental da cidade de São Paulo. [dissertação]. São Paulo (SP): Faculdade São Camilo de Administração Hospitalar; 1991.

15. Anselmi ML, Angerami ELS, Ribas Gomes EL. Rotatividade e condições de trabalho em enfermagem nos hospitais do Município de Ribeirão Preto. Rev Bras Saúde Ocupac 1997; 23(85/86):31-41. 
16. Anselmi ML, Angerami ELS, Ribas Gomes EL. Rotatividade dos trabalhadores de enfermagem nos hospitais do Município de Ribeirão Preto. Rev Panam Salud Publica 1997 julho; 2(1):44-50.

17. Bezerra ALQ. O significado da rotatividade de pessoal numa instituição de saúde privada. Rev Bras Enfermagem 1997 janeiro/ março; 50(1):107-20.

18. Souza JMP de Uso da técnica de tábua de sobrevivência para estimar sobrevida em casos de câncer. Rev Bras Cancerol 1976; 26(1):57-9.

19. Pugliese C, Lessa I, Santos A Filho. Estudo da sobrevida na miocardite crônica de chagas descompensada. Rev Inst Med Trop 1976 maio/junho; 18(3):191-201.
20. Laurenti R, Mello Jorge MHP, Lebrão ML, Gotlieb SLD. Estatísticas de saúde. $2^{\mathrm{a}}$ ed. São Paulo (SP): EPU; 1987.

21. Collett $D$. Modelling survival data in medical research. London (UK): Chapman \& Hall; 1994.

22. Lane KF, Andrew JE. A method of labour turnover analysis. J $R$ Stat Soc 1955; 118(Pt 3): 296-314.

23. Pereira MJB, Fortuna CM, Silva EM, Pinto IC, Mishima SM, Almeida MCP. Enfermeiros na rede básica de Ribeirão Preto e as políticas de saúde - situação atual. $5^{\circ}$ Congresso Brasileiro de Saúde Pública e $5^{\circ}$ Congresso Paulista de Saúde Coletiva; 25-29; 1997. Águas de Lindóia: São Paulo (SP); 1997. 